NASA Technical Memorandum 81463

NASA-TM-81463 19800010134

ANALYSIS AND DESIGN OF A UNIFORM-CLEARANCE, PUMPING-RING ROD SEAL FOR THE STIRLING ENGINE

\title{
I. Etsion
}

Lewis Research Center

Cleveland, Ohio

March 1980 
ANALYSIS AND DESIGN OF A UNIFORM-CLEARANCE,

PUMPING-RING ROD SEAL FOR THE STIRLING ENGINE*

by 1. Etsion

National Aeronautics and Space Administration

Lewis Research Center

Cleveland, Ohio

SUMMARY

A uniform-clearance pumping ring, as opposed to the conventional

taper-clearance one, is described. The uniform-clearance concept

eliminates complex elastohydrodynamic problems and enables a simple

analytical treatment to be made. An analytical expression is derived

for the pumping rate showing the effect of varicus design parameters on

the pumping ring's performance. An optimum cle rance is found by which

the pumping rate is maximized and a numerical e ample is presented to

demonstrate the potential of the uniform-clearance design.

INTRODUCTION

Due to the energy crisis of the 1970's, a great deal of interest

has been shown in development of the Stirling engine as an alternate to

* The research described in this report was done while the author was a National Research Council - NASA Resea-ch Associate at the NASA Lewis Research Center. 
the internal combustion engine. One of the major problems in the stiritig engine development is the sealing of thi high prissure working fluid (ref. 1). Towards this end several recipr cating suals are used, with various amount of success, including a pump ng ring which forms the external seal (ref. 2). The pumping ring (fig. 1) is a cylindrical bushing forced by some means against the reciprocating rod. The ring is either pressed conically or given an inside radial taper to provide a converging gap in the pumping diruction. The inside radial taper is considered to be the key factor for successful operation of the pumping ring. As the rod translates in the pumping direction, large hydrodynamic pressures are developed in the thin tapered fluid film between the ring and the rod. The hydrodynamic forces expand the ring and allow fluid to be dragged through the clearance space. On the return stroke, the hydrodynamic effect reduces the pressure allciving th? ring to scrape on the rod and prevent fluid from leaking back.

Performance analysis of the pumping ring described above is a formidable task. The pressures developed in the claarance setween the ring 
and the rod depend on the shape of the clearance. However, due to ring elasticity the clearance depends on the hydrodynamic pressure generated by the rod motion. Hence, the problem is an elastohydrodynamic one for which the hydrodynamic and elasticity governing equations have to be solved simultaneously. Only few attempts have been made so far to solve this difficult problem (refs. 3 to 6 ). These solution methods tend to be either restrictive or very complex.

The essence of pumping effect in any pumping-ring seal is simply a cyclic clearance variation which permits large amount of viscous-shear flow during the pumping stroke and restricts the leakage during the return stroke. Hence, the key factor in pumping ring design should be the proper clearance variation rather than the means by which it is achieved. The radial taper pumping ring utilizes a relatively simple hydrodynamic mechanism to generate the clearance variation. Unfortunately, this simplicity is overridden by the complexity of calculating clearance variation, making performance prediction very quistionabie.

This paper presents the analysis and design of a uniform-clearance pumping ring. The clearance variation is accomplished through means 
other than hydrodynamic pressures, hence, eliminating the complexity of the elastohydrodynamic problem. One possible mechanism is shown in Figure 2 where an annular cavity on the back of the ring is properly pressurized in some cyclic mode synchronized with the rod motion. By maintaining a uniform radial clearance between the ring and the rod, and thus eliminating the hydrodynamic pres;ures, the design and performance analysis of the pumping $r$ ing become simple.

NOMENCLATURE
a rod stroke
C radial clearance
$c_{\text {in }}$ initial clearance
$c_{p}^{*}$ optimum clearance, equation (8)
E modulus of elasticity of ring material
h ring wall thickness
$L \quad$ ring length
$P$ preload maximum pressure 


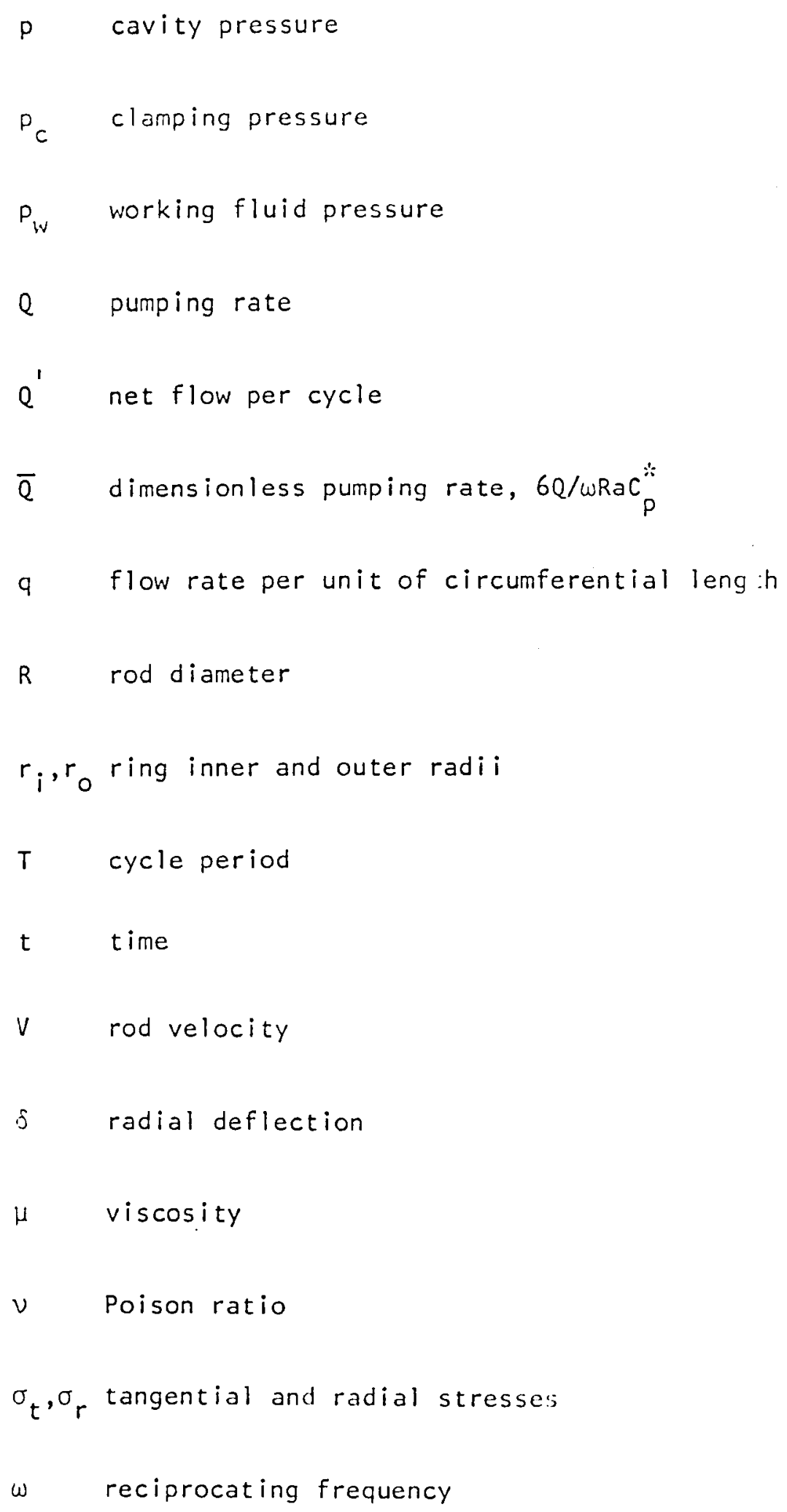




\section{Subscripts:}

$$
\begin{aligned}
& \text { p pumping stroke } \\
& r \quad \text { return stroke }
\end{aligned}
$$

\section{PUMPING RATE ANALYSIS}

Consider the pumping ring of Figure 2 , the instantaneous flow rate per unit of circumferential length into the high pressure region is:

$$
q= \pm v \frac{c}{2}-\frac{c^{3}}{12 \mu} \frac{P_{w}}{L}
$$

where the $+V$ and $-V$ are for the pumping stroke or the return stroke, respectively. Designating the pumping and return strokes by the subscripts $p$ and $r$, respectively, the clearance during the pumping stroke will be $c_{p}$ and during the return stroke $c_{r}$. The net flow into the high pressure side is

$$
Q^{\prime}=2 \pi R \int_{0}^{T / 2}\left(q_{p}+q_{r}\right) d t
$$

where $T$ the cycle period is

$$
T=\frac{2 \pi}{\omega}
$$

and $w$ is the reciprocating frequency. Substititing equation (1) in

equation (2) and noting that 


$$
-\int_{0}^{T / 2} v d t=a
$$

where $a$ is the rod stroke. The net flow into the high pressure side

during one complete cycle i:

$$
Q^{\prime}=2 \pi R\left[\frac{a}{2}\left(c_{p}-c_{r}\right)-\frac{P_{w}}{12 \mu L} \frac{T}{2}\left(c_{p}^{3}+c_{r}^{3}\right)\right]
$$

Dividing equation (5) by the period $T$ and substituting equation (3),

we finally have the pumping rate in the form

$$
Q=\frac{\omega R}{2}\left[a\left(c_{p}-c_{r}\right)-\frac{\pi P_{w}}{6 \mu L \omega}\left(c_{p}^{3}+c_{r}^{3}\right)\right]
$$

An optimum clearance $c_{p}$ can be found which will maximize the pumping rate for any preselected $C_{r}$. Differentiating equation (6) with respect to $C_{p}$ and equating to zero we find

$$
a-\frac{\pi p_{w}}{2 \mu L \omega} c_{p}^{2}=0
$$

thus

$$
c_{p}^{*}=\left(\frac{2 \mu L \omega a}{\pi p_{w}}\right)^{1 / 2}
$$

Equation (8) provides the optimum pumping-stroke clearance $C_{p}$, which ensures maximum pumping rate for the pumping ring of a specified $C_{r}$.

This optimum clearance depends on the stroke, a, and reciprocating frequency, $w$, of the rod; on the length, $L$, of the pumping $r i n g ;$ and on the 
viscosity, $\mu$, and pressure, $p_{w}$, of the working fluid. It is clear from equation (6) that a clearance $C_{p}$ too small or too large can alter drastically the pumping rate. If $C_{p}$ is close to $C_{r}$, the pressure induced leakage can overcome the viscous shear pumping and the high pressure oil leaks through the gap. Also, when increasing $C_{p}$ the pressure induced leakage increases as a cubic function of $C_{p}$ while the viscous shear pumping increases only linearly. Hence, a value of $c_{p}$ may be reached where again the high pressure oil leaks to the low pressure side. The preceding discussion demonstrates the importance of an accurately controlled pumping-stroke clearance, $C_{p}$. It is difficult to achieve fine control by hydrodynamic pressures associated with the tapered pumping ring design (refs. 3 and 4 ). In fact, the hydrodynamic force may even expand the ring too much thereby destruying the shole sealing effect.

Equation (6) can be arranged in the. form

$$
Q=\frac{R}{2} \omega a C_{p}\left\{1-\frac{C_{r}}{C_{p}}-\frac{\pi p_{w}}{6 \mu L \omega a} c_{p}^{2}\left[1+\left(\frac{c_{r}}{C_{p}}\right)^{3}\right]\right\}
$$

which after using the relation 


$$
c_{p}=c_{p}^{\therefore} \frac{c_{p}}{c_{p}^{* i}}
$$

and substituting equation ( 8 ) becomes

$$
Q=\frac{\omega R a C_{p}^{*}}{6} \bar{Q}
$$

where $\bar{Q}$, the dimensionless pumping rate, is

$$
\bar{Q}=3 \frac{c_{p}}{c_{p}^{*}}-\left(\frac{c_{p}}{c_{p}^{*}}\right)^{3}-3 \frac{c_{r}}{c_{p}^{*}}-\left(\frac{c_{r}}{c_{p}^{*}}\right)^{3}
$$

Equation (11) can be expressed in terms of all the design parameters

again using equation ( 8 ), hence,

$$
Q=\frac{R}{\sigma}(w a)^{3,2}\left(\frac{2 \mu L}{\pi P_{w}}\right)^{1 / 2} \bar{Q}
$$

As can be seen from equation (13), the main factors affecting the pumping rate are the rod frequency $\omega$ and stroke $a$, and the ratios $C_{p} / C_{p}^{*}$ and $C_{r} / C_{p}^{*}$. These two clearance ratios are actually the essence of the uniformclearance pumping-ring design. From equation (13) it is clear that in order to have a positive pumping rate the dimensionless parameter $\vec{Q}$ must be positive; that is, 


$$
3 \frac{c_{p}}{c_{p}^{* 1 *}}-\left(\frac{c_{p}}{c_{p}^{*}}\right)^{3}-3 \frac{c_{r}}{c_{p}^{*}}-\left(\frac{c_{r}}{c_{p}^{*}}\right)^{3}>0
$$

For an optimum design when $C_{p}=c_{p}^{*}$ the solution ( $f$ equation (14) gives

$$
\frac{c_{r}}{c_{p}^{*}}<0.596
$$

Hence, in addition to designing an optimum pumping.stroke clearance $c_{p}^{*}$, as given by equation $(8)$, the return-stroke clearance $c_{r}$ should be limited by $c_{r}<0.596 c_{p}^{*}$.

The maximum pumping rate can be achieved (see eqs. (12) and (13)) when $c_{p}=c_{p}^{*}$ and $c_{r}=0$. However, to avoid rutbing which can result in excessive friction loss and wear, it is preferable to operate the pumping ring with a ratio $C_{r} / C_{p}^{*}>0$. Figure 3 presents the effect of the return-stroke clearance ratio $C_{r} / c^{\prime}$, on the pumping rate. As can be seen from Figure 3 , the ultimate maximum pumping rate $\bar{Q}$ corresponding to $C_{r} / C_{p}^{*}=0$ and $C_{p} / C_{p}^{*}=1$ is $\bar{Q}=2$. Increasing the return-stroke clearance ratio to $c_{r} / c_{p}^{*}=0.1$ reduces the max mum pumping rate by only 15 percent to $\bar{Q}=1.7$. 


\section{DEFORMATION ANALYSIS}

As was shown in the previous sections, the pumping capacity of the uniform-clearance $r i n g$ is based on maintaining different radial clearances during the pumping and return strokes. To that end, the ring has to be deflected so as to create this necessary difference. If initially the radial clearance between the ring and rod is $c_{\text {in }}$ then the required radial deflections $\delta_{p}$ and $\delta_{r}$ corresponding to the pumping and return strokes, respectively, are

$$
\begin{aligned}
& \delta_{p}=c_{p}-c_{\text {in }} \\
& \delta_{r}=c_{r}-c_{\text {in }}
\end{aligned}
$$

A radial deflection of the ring can be achieved by subjecting it to a uniform external pressure (see fig. 2). Under such conditions the tangential and radial stresses at the inner radius of the $r i n g, r_{i}$, are

$$
\sigma_{t}=-\frac{2 p r_{0}^{2}}{r_{0}^{2}-r_{i}^{2}}
$$

and

$$
\sigma_{r}=0
$$


The radial deflection is

$$
\frac{\dot{0}}{r}=\frac{\sigma_{t}}{E}-\nu \frac{\sigma_{r}}{E}
$$

Hence, by equations (17), (18) and (19) the radial deflection of the

inner radius of the ring is

$$
\delta=-\frac{p}{E} \frac{2 r_{0}^{2} r_{i}}{r_{0}^{2}-r_{i}^{2}}
$$

Using equations (15), (16) and (20) we can calculate the required cavity pressures $p_{p}$ and $p_{r}$ for the pumping and returin strokes, respectively. Thus,

$$
\begin{aligned}
& P_{p}=\frac{E C_{p}}{2 r_{i}}\left(\frac{C_{i n}}{C_{p}}-1\right)\left[1-\left(\frac{r_{i}}{r_{0}}\right)^{2}\right] \\
& p_{r}=\frac{E C_{p}}{2 r_{i}}\left(\frac{C_{i n}}{C_{p}}-\frac{C_{r}}{C_{p}}\right)\left[1-\left(\frac{r_{i}}{r_{0}}\right)^{2}\right]
\end{aligned}
$$

From equations (21) and (22) it can be seen that the cavity pressures are mostly affected by the ratios $C_{i n} / C_{p}, c_{r} / C_{p}$ and $r_{i} / r_{0}$. The required pressures can be reduced by making $C_{i n}=C_{p}$; in which case the pumpingstroke cavity pressure is zero, and the return-siroke cavity pressure is 


$$
p_{r}=\frac{E C_{p}}{2 r_{i}}\left(1-\frac{C_{r}}{C_{p}}\right)\left[1-\left(\frac{r_{i}}{r_{0}}\right)^{2}\right]
$$

When the engine is not running the pumping : ing has to function as a static seal. This means that the ring has to bt clamped on the rod so that the clearance between them becomes zero or there is even an interference fit. To achieve this, the cavity pressure during stand still has to be increased to a value that will make $\delta>-C_{\text {in }}$. Hence, from equation (20) and for $C_{i n}=C_{p}$

$$
P_{c}>\frac{E C_{p}}{2 r_{i}}\left[1-\left(\frac{r_{i}}{r_{o}}\right)^{2}\right]
$$

For simplicity of the pressurization system, the working-fluid pressure, $P_{w}$, can serve as the clamping pressure $P_{c}$ provided that $P_{w}>P_{c}$. The preceding deflection analysis was based on the assumption that the ring has a plain cylindrical bore. However, after assembling in the engine, an initially plain cylindrical ring is d:storted by the internal hydrostatic pressure distribution as shown in $F$ gure $4(a)$. Thus, to ensure a plain cylindrical bore after assembly, the ring must be preloaded 
by an external load that produces an equal, but cpposite, deflection to that generated by the internal pressure. Since the internal pressure is not uniform but drops linearly from $P_{w}$ to zero, the external load would have to result in a similar linear drop from some value $P$ to zero as shown in Figure $4(\mathrm{~b})$. The pressure $P$ can be calculated from the requirement of zero radial deflection at the inner radius of the ring under the combined action of both $P$ and $P_{W}$. If $P$ and $\mathrm{p}_{\mathrm{w}}$ were uniform, the tangential and radial stresses of the ring inner radius would be (ref. 7)

$$
\begin{aligned}
& \sigma_{t}=\frac{p_{w} r_{i}^{2}-p r_{0}^{2}-\left(p-p_{w}\right) r_{0}^{2}}{r_{0}^{2}-r_{i}^{2}} \\
& \sigma_{r}=\frac{p_{w} r_{i}^{2}-p r_{0}^{2}+\left(p-p_{w}\right) r_{0}^{2}}{r_{0}^{2}-r_{i}^{2}}
\end{aligned}
$$

Substituting equations (25) and (26) in equation (19) and requiring

$\delta=0$ at $r=r_{i}$, we find

$$
\left(p_{w} r_{i}^{2}-p r_{0}^{2}\right)(1-v)-\left(p-p_{w}\right) r_{0}^{2}(1+v)=0
$$

or

$$
p=\frac{p_{w}}{2}\left[1+v+\left(\frac{r_{i}}{r_{0}}\right)^{2}(1-v)\right]
$$


The linear drop of the external load from $P$ at the high pressure side to zero at the low pressure side can be obtained by means of a conical press or shrink fit. In this case the outer radius, $r_{0}$, in equations (17) through (24) is that of the external member pressed or shrink fitted on the ring.

NUMERICAL EXAMPLE

The following numerical data is used for a pumping ring design example

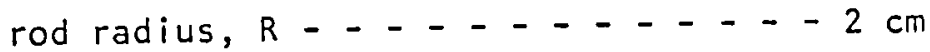
rod stroke, a - - - - - - $5 \mathrm{~cm}$ ring length, $\mathrm{L} \ldots-\ldots-{ }_{-} .5 \mathrm{~cm}$ engine frequency, $\omega \ldots \ldots-\ldots$ - $377 \mathrm{rad} / \mathrm{sec}(3600 \mathrm{rpm})$ oil pressure, $p_{\mathrm{w}}-\ldots-\ldots \mathrm{MPa}$ oil viscosity, $\mu-\ldots-\ldots$ - - 5 mPa.s ( AE 30 at $112^{\circ} \mathrm{C}$ ) ring modulus of elasticity, E - - 111 GPA (brinze) The optimum pumping stroke clearance is calculated from equation (8) and is 


$$
c_{p}^{*}=12.25 \mu \mathrm{m}
$$

Selecting a clearance ratio $C_{r} / C_{p}^{*}=0.1$ the puriping rate is found from equations (11) and (12)

$$
Q=1.31 \mathrm{~cm}^{3} / \mathrm{sec}=78.50 \mathrm{~cm}^{3} / \mathrm{min}
$$

Selecting initial clearance $c_{i n}=c_{p}^{*}$ the required cavity pressure during the pumping stroke is (eq. (21)) $p_{p}=0$. The cavity pressure required for $c_{r}=0.1 c_{p}^{*}$ during the return stroke is calculated from equation (23) and the clamping pressure $p_{c}$ from equation (24). Table I presents values of the pressures $p_{r}$ and $p_{c}$ calculated for various wall thickness values of the composite ring. As can be seen from Table 1 a ring having wall thickness of $3 \mathrm{~mm}$ will require a cavity pressure $p_{r}=7.5 \mathrm{MPa}$ during the return stroke, and a clamping pressure $p_{c}=$ 8.3 MPa (which is less than $\mathrm{p}_{\mathrm{w}}$ ) when the engine is not running.

The ring can be made by press-fitting an outer member with wall thickness of $1 \mathrm{~mm}$ on an inner member having a wall thickness of $2 \mathrm{~mm}$. The conical press fit will require a maximum pressure $P$ which, by equation (27) and for an inner member radius ratio of $r_{i} / r_{0}=20 / 22$, is 


$$
P=9.4 \mathrm{MPa}
$$

The composite ring is subjected to an oscillating load because $p_{r}$ is being applied at the enyine frequency. The maximum tangential stress

generated by $P_{r}$ at the inner radius of the ring is, by equation (17),

$$
\sigma_{t}=-2 p_{r} \frac{r_{o}^{2}}{r_{0}^{2}-r_{i}^{2}}
$$

Using the values $P_{r}=7.5 \mathrm{MPa}, r_{i}=2 \mathrm{~cm}$, and $r_{0}=2.3 \mathrm{~cm}$, we find from equation (28)

$$
\sigma_{t}=-61.5 \mathrm{MPa}
$$

In addition to this repeated stress, the inner mamber of the ring is loaded by $P$ and $P_{W}$. The maximum stresses at the inner radius due to these pressures are calculated from equation: (25) and (26) where now $r_{0}=2.2 \mathrm{~cm}$. The result is

$$
\begin{aligned}
& \sigma_{t}=-16 \mathrm{MPa} \\
& \sigma_{r}=-7 \mathrm{MPa}
\end{aligned}
$$

Hence, the tangential stress amplitude is $-30.75 \mathrm{MPa}$ and the nean tangential stress is $-44.75 \mathrm{MPa}$. The radial stress amplitude is zero and the mean radial stress is $-7 \mathrm{MPa}$. The mean and alternating 
Von Mises stresses are, therefore (ref. 8) $48.6 \mathrm{MPa}$ and $7 \mathrm{MPa}$, respectively. Available fatigue test data of copper-base alloys indicate bending fatigue-strength to tensile-strength ratio of about 0.35 which for hard bronze means fatigue strength of about $145 \mathrm{MPa}$. Since the pumping ring is under compression, it seems that the design is safe.

RESULTS AND DISCUSSION

The optimum design resulted in a pumping-stroke clearance $c_{p}^{*}=$ $12.25 \mu \mathrm{m}$. For this clearance a pumping rate $Q=78.5 \mathrm{~cm}^{3} / \mathrm{min}$ can be obtained when the return-stroke cavity pressure is $p_{r}=7.5 \mathrm{MPa}$ which ensures clearance ratio $c_{r} / c_{p}^{*}=0.1$

Any change in $\mathrm{Pr}_{\mathrm{r}}$ will, of course, change the pumping rate due to the effect of $P_{r}$ on the clearance ratio $c_{r} \cdot C_{p}^{*}$. The pumpingstroke clearance $C_{p}$ may also differ from the optimum value of $c_{p}^{*}$ due to manufacturing tolerances. Hence, it is of interest to examine off-design pumping rates for various values of umping-stroke clearances $C_{p}$ and return-stroke cavity pressures ' $r$ ' For an easy 
comparison equations (11), (12) and (23) can be rearranged in the form

$$
Q=\frac{\omega \operatorname{RaC} C_{p}^{*}}{6}\left[3 \frac{{ }^{c}}{c_{p}^{*}}-\left(\frac{c_{p}}{c_{p}^{*}}\right)^{3}-3 \frac{c_{r}}{c_{p}^{*}}-\left(\frac{c_{r}}{c_{p}^{*}}\right)^{3}\right]
$$

and

$$
\frac{c_{r}}{c_{p}^{* \frac{k}{*}}}=\frac{c_{p}}{c_{p}^{* *}}-\frac{2 r_{i} p_{r}}{E C_{p}^{*}\left[1-\left(\frac{r_{i}}{r_{0}}\right)^{2}\right]}
$$

Where $c_{p}^{*}$ is the optimum pumping-stroke clearance given in equation (8) as

$$
c_{p}^{*}=\left(\frac{2 \mu L \omega a}{\pi P_{w}}\right)^{1 / 2}
$$

For any ratio $i_{p} / C_{p}^{*}$, equation (33) is used first to calculate $c_{r} / C_{p}^{*}$ as function of both $c_{p} / c_{p}^{*}$ and $p_{r}$. The result is then used in equation (32) to calculate the pumping rate.

Figure 5 presents pumping rates, for the pumping ring of the numerical example, as a function of the cavity pressure $P_{r}$ at various pumping-stroke clearance values. The clearances $C_{p}$ and $C_{r}$ are normalized by $c_{p}^{*}$ where $c_{p}^{*}=12.25 \mu \mathrm{m}$. As can be seen from Figure 5 , at each given pumping-stroke clearance, $C_{p}$, there are two distinct 
cavity pressures $P_{r}$ corresponding to the cases $Q=0$ and $C_{r}=0$, respectively. If the return-stroke cavity pressule, $p_{r}$, is below the minimum value for $Q=0$, then the ratio of the return-stroke to pumping-stroke clearances $C_{r} / C_{p}$ becomes too large, the pressure induced flow overcomes the viscous-shear pumping and the working fluid starts leaking through the clearance gap. Increasing the pressure $\mathrm{P}_{r}$ reduces the return-stroke clearance $C_{r}$ and, therefore, the clearance ratio $C_{r} / c_{p}$. The result is an increase in the pumping rate. The pressure $P_{r}$ and, hence, the pumping rate can be increased up to the point where $C_{r}$ vanishes. At this point the clamping pressure $p_{c}$ is reached and further increase of $\mathrm{P}_{r}$ will not affect the pumping rate.

The benefit of optimizing the pumping-stroke clearance is obvious. Trying to keep a pumping rate of $78 \mathrm{~cm}^{3} / \mathrm{min}$, which in the design example is obtained when $c_{p} / c_{p}^{*}=1$ and $c_{r} / c_{p}^{*}=0.1$, becomes impossible if the ratio $C_{p} / C_{p}^{*}$ is either reduced or increased by about 30 percent with respect to its optimum.

Figure 5 can be used as a design chart for pumping rings activated 
by external pressure and having the design requirement of the example. Similar charts can, of course, be plotted for different designs. However, for a more general use one would prefer to use Figure 3. As was mentioned in the Introduction, the essence of any pumping ring is the cyclic variation of its clearance rather than the means by which this variation is achieved. Hence, the pumping rate depends completely on the ratios $C_{p} / C_{p}^{*}$ and $C_{r} / c_{p}^{*}$ regardless of the mechanism causing the ring deflection. Once the designer has used equation (8) to calculate $c_{p}^{*}$ and he has any possible mean to control $c_{p}$ and $c_{r}$, Figure 3 can be used to determine pumping rates at any known combination of the ratios $C_{p} / C_{p}^{*}$ and $C_{r} / C_{p}^{*}$

An important result of Figure 3 is the lower and upper limits of $c_{p} / c_{p}^{*}$ at given ratios of $c_{r} / c_{p}^{*}$. At $c_{r}=0$, for example, $c_{p} / c_{p}^{*}$ for a positive pumping rate is 1 imited by the value $C_{p} / C_{p}^{\star}<\sqrt{3}$. As $c_{r} / C_{p}^{*}$ increases the range of permissible deviation from the optimum design $c_{p}=c_{p}^{*}$ is narrowing down. At $c_{r} / c_{p}^{*}=0.3$, fo- example, the operating pumping-stroke clearance must be greater than $0.3 c_{p}^{*}$ but 
less than $1.55 \mathrm{C}_{\mathrm{p}}^{*}$. This result underlines the importance of controlling the pumping and return-strokes clearances and contradicts the assumption presented in a recently published work (ref. 9) that the higher the value of $C_{p}$, the better. In fact in the hydrodynamic pressure activated ring the pumping-stroke clearance may become too large causing failure due to leakage.

CONCLUDING REMARKS

A controlled cyclic variation of pumping ring clearance is the key factor in its successful operation. The radial taper pumping ring utilizes a relatively simple mechanism to cause clearance variation, however, this mechanism involves complex elastohydrodynamic effects. The uniform-clearance pumping ring, on the other hand, needs a somewhat more elaborate auxiliary mechanism but its performance analysis is straightforward.

A uniform-clearance pumping ring for the Stirling engine was analyzed and its capacity demonstrated through a design example. It was found that pumping rates can be maximized by optimization of the 
pumping-stroke clearance. It was also found tha: if the ratio of return-stroke over pumping-stroke clearance excesds a critical

value or if the pumping-stroke clearance is too high, the pumping ring starts to leak.

An external pressure variation synchronized with the rod motion . was suggested for the design example. However, any other means that can serve the purpose of controlling cyclic clearance variation will do as well. 


\section{REFERENCES}

1. Rietdijk, J. A., et al.: A Positive Rod or Piston Seal for Large

Pressure Differences. Philips Tech. Rev., Vol. 26, N. 10, 1965, pp. $287-296$.

2. Theeuwes, G.: Dynamic High-Pressure Seals in Stirling Engines.

Proc. 8th Int. Conf. on Fluid Sealing, Sept. 1978, Paper Jl.

Published by BHRA.

3. Kuzma, D. C.: Analysis of Pumping Rings. Jour. of Lub. Tech., Trans ASME, Vol. 93, No. 2, April 1971, pp. 287-292.

4. Zull, L. M.; and Kettleborough, C. F.: An Elastohydrodynamic Analysis of Transient Pumping Ring Operatiou. Jour. of Lub. Tech., Trans ASME, Vol. 97, No. 2, April 1575, pp. 195-201.

5. Ruskell, L.E. C.: Reynolds Equation and Elastohydrodynamic Lubrication in Metal Seals. Proc. R. Soc., London A. 399 , 1976, pp. 383-396.

6. Smith, P. J.: A Numerical Simulation of the Stirling Engine $0 i 1$ Pumping Ring Seal. M.Sc. Thesis, The University of Toledo, June 1979. 
7. Wang, C.: Applied Elasticity, McGraw-Hill Brok Compony, 1953.

8. Shigley, J. E.: Mechanical Engineering Design, 3rd edition, McGraw-Hill Book Company, 1977.

9. Ruskell, L. E. C.: The Elastohydrodynamic Performance of LowFriction, Zero-Leakage Metal Seals. Jour. of Mech. Engng. Sci., Vol. 21, No. 4, 1979, pp. 275-285. 
Table 1. - Return Stroke Cavity Pressure $P_{r}$ and Clamping Pressure $p_{c}$ for Various Wall Thickness Values $h$

\begin{tabular}{|c|c|c|}
\hline $\begin{array}{c}\text { Wall } \\
\text { thickness } \\
\text { h mm }\end{array}$ & $\begin{array}{c}\text { Return stroke } \\
\text { cavity pressure } \\
\mathrm{P}_{\mathrm{MPa}}\end{array}$ & $\begin{array}{c}\text { Clamping } \\
\text { pressure } \\
\mathrm{P}_{\mathrm{c}} \text { MPa }\end{array}$ \\
\hline 2 & 5.3 & 5.9 \\
3 & 7.5 & 8.3 \\
4 & 9.3 & 10.6 \\
5 & 11.0 & 12.2 \\
\hline
\end{tabular}




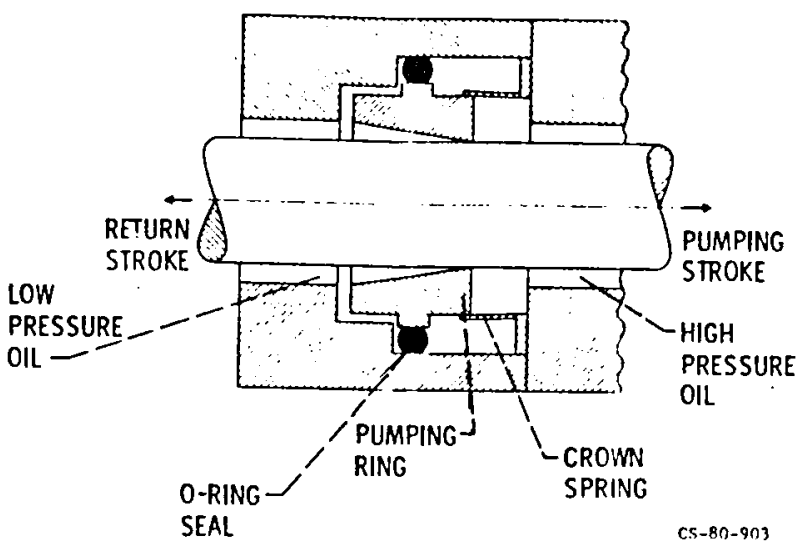

Figure 1. - Pumping ring assembly.

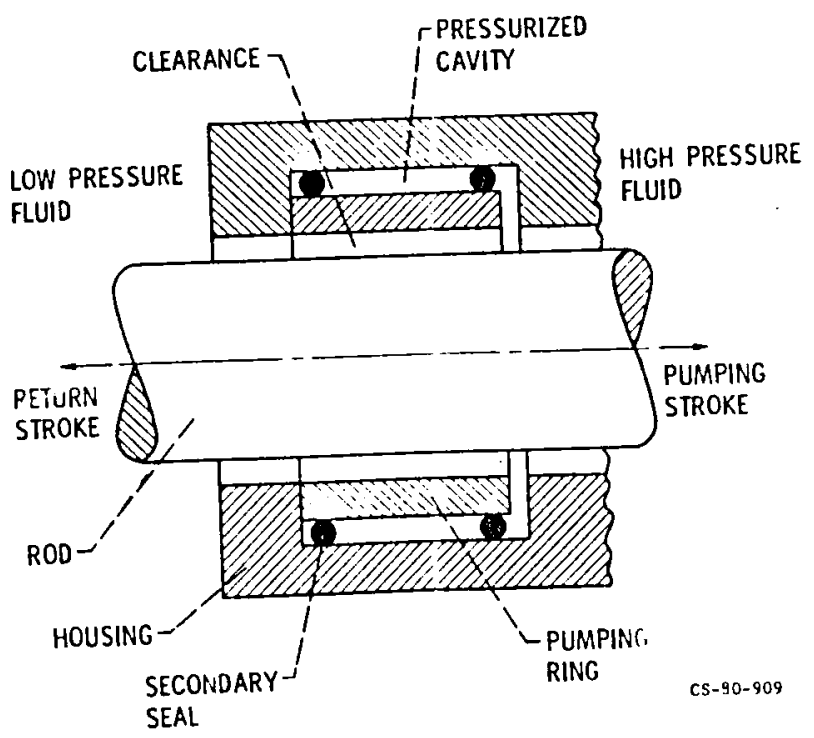

Figure 2. Uniform-clearance pumping ring. 


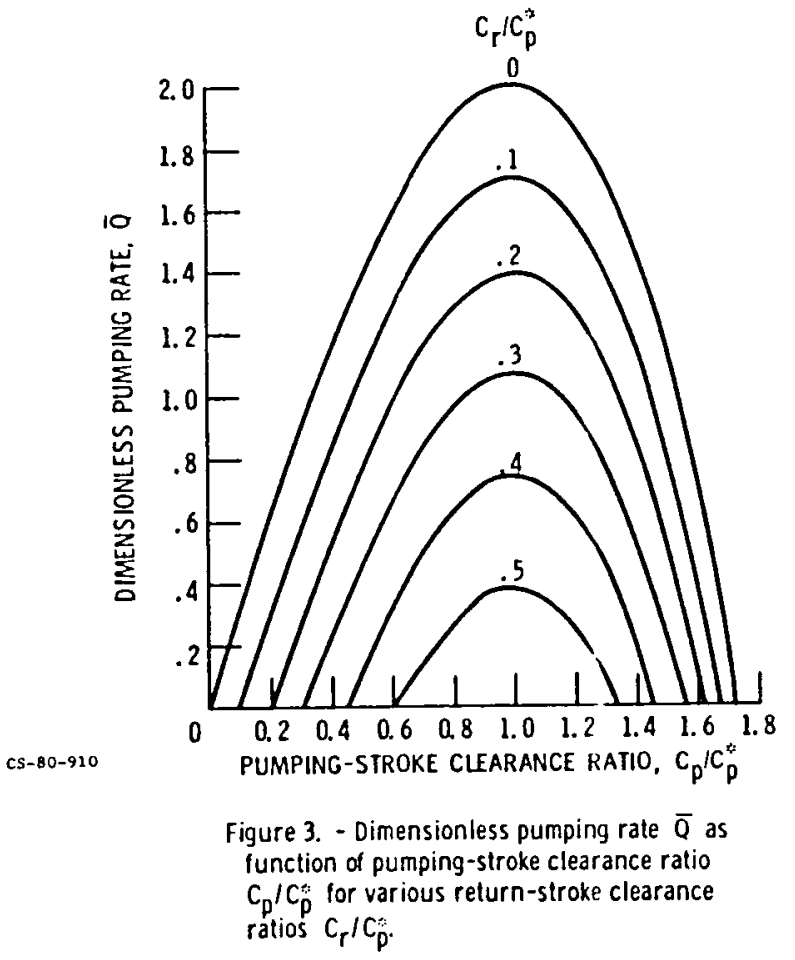



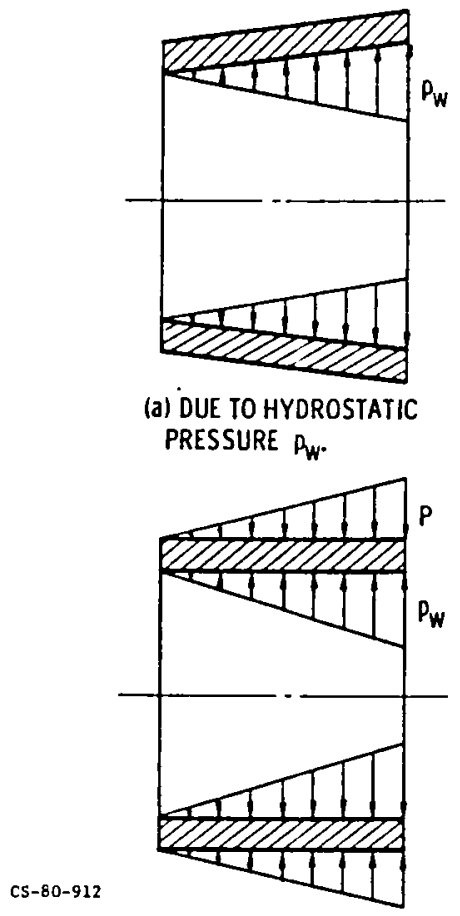

(b) DUE TO COMBINED HYDRO-

STATIC PRESSURE $P_{W}$ AND

PRELOAD P.

Figure 4. - Cylindrical ring deflection.

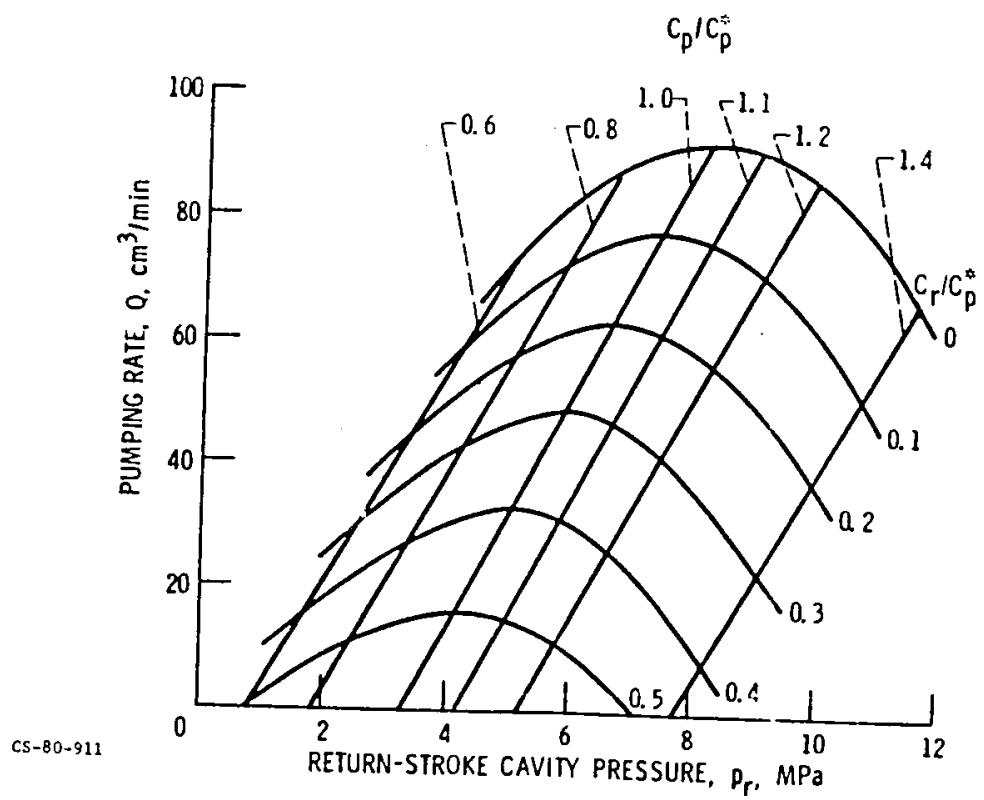

Figure 5. - Pumping rate, $Q$, as function of cavity pressure, $P_{r}$, for various clearance values, $C_{p} / C_{p}$. 


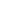




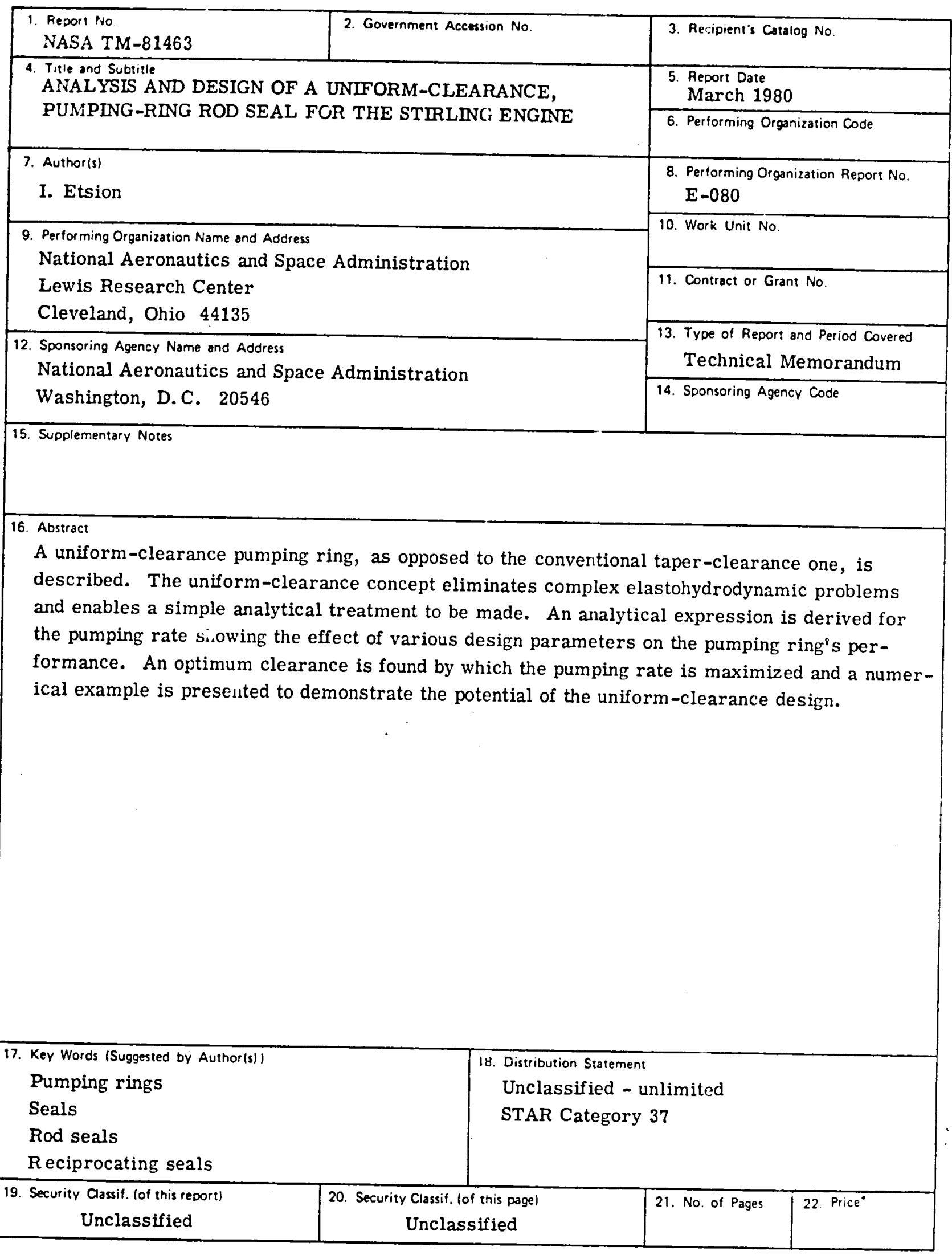

"For sale by the National Technical Information Service. Springfield. Virginia 22161 

National Aeronautics and Space Administration

Washington, D.C.

20546

Official Business

Penalty for Private Use, $\mathbf{\$ 3 0 0}$
SPECIAL FOURTH CLASS MAIL BOOK

Postage and Fees Paid National Aeronautics and Space Administration NASA-451

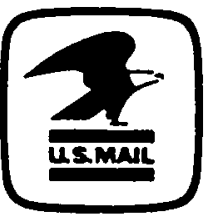

